\title{
Richard Poulin
}

Sociologue, professeur titulaire, département de sociologie et d'anthropologie, Université d'Ottawa.

\section{(2005)}

\section{ÉDITORIAL:}

\section{"Quinze thèses sur le capitalisme et le système prostitutionnel mondial."}

Un document produit en version numérique par Jean-Marie Tremblay, bénévole, professeur de sociologie retraité du Cégep de Chicoutimi

Courriel: jean-marie_tremblay@uqac.ca

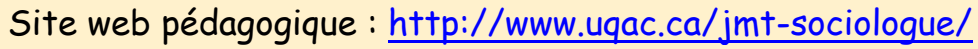

Dans le cadre de: "Les classiques des sciences sociales"

Une bibliothèque numérique fondée et dirigée par Jean-Marie Tremblay, professeur de sociologie au Cégep de Chicoutimi

Site web: http://classiques.uqac.ca/

Une collection développée en collaboration avec la Bibliothèque

Paul-Émile-Boulet de l'Université du Québec à Chicoutimi Site web: $h+t p: / /$ bibliotheque.uqac.cal 


\section{Politique d'utilisation de la bibliothèque des Classiques}

Toute reproduction et rediffusion de nos fichiers est interdite, même avec la mention de leur provenance, sans l'autorisation formelle, écrite, du fondateur des Classiques des sciences sociales, Jean-Marie Tremblay, sociologue.

Les fichiers des Classiques des sciences sociales ne peuvent sans autorisation formelle:

- être hébergés (en fichier ou page web, en totalité ou en partie) sur un serveur autre que celui des Classiques.

- servir de base de travail à un autre fichier modifié ensuite par tout autre moyen (couleur, police, mise en page, extraits, support, etc...),

Les fichiers (.html, .doc, .pdf, .rtf, .jpg, .gif) disponibles sur le site Les Classiques des sciences sociales sont la propriété des Classiques des sciences sociales, un organisme à but non lucratif composé exclusivement de bénévoles.

Ils sont disponibles pour une utilisation intellectuelle et personnelle et, en aucun cas, commerciale. Toute utilisation à des fins commerciales des fichiers sur ce site est strictement interdite et toute rediffusion est également strictement interdite.

L'accès à notre travail est libre et gratuit à tous les utilisateurs. C'est notre mission.

Jean-Marie Tremblay, sociologue

Fondateur et Président-directeur général, LES CLASSIQUES DES SCIENCES SOCIALES. 
Cette édition électronique a été réalisée par Jean-Marie Tremblay, bénévole, professeur de sociologie au Cégep de Chicoutimi à partir de :

\section{Richard POULIN}

"Quinze thèses sur le capitalisme et le système prostitutionnel mondial". ÉDITORIAL.

Un article publié dans la revue Alternatives Sud, vol. 12, no 3, automne 2005, pp. 7-29. "Éditorial". Numéro intitulé : "Prostitution, la mondialisation incarnée." Un numéro coordonné par Richard Poulin. Paris : Centre tricontinental et Syllepse.

[Autorisation formelle accordée par l'auteur le 13 septembre 2011 de diffuser ce texte dans Les Classiques des sciences sociales.]

Courriel : Richard.Poulin@uottawa.ca

Polices de caractères utilisée : Comic Sans, 12 points.

Édition électronique réalisée avec le traitement de textes Microsoft Word 2008 pour Macintosh.

Mise en page sur papier format : LETTRE US, $8.5^{\prime \prime} \times 11^{\prime \prime}$

Édition numérique réalisée le 4 octobre 2011 à Chicoutimi, Ville de Saguenay, Royaume du Saguenay, Québec. 


\section{Richard Poulin}

Sociologue, professeur titulaire, département de sociologie et d'anthropologie, Université d'Ottawa.

\section{"Quinze thèses sur le capitalisme et le système prostitutionnel mondial"}

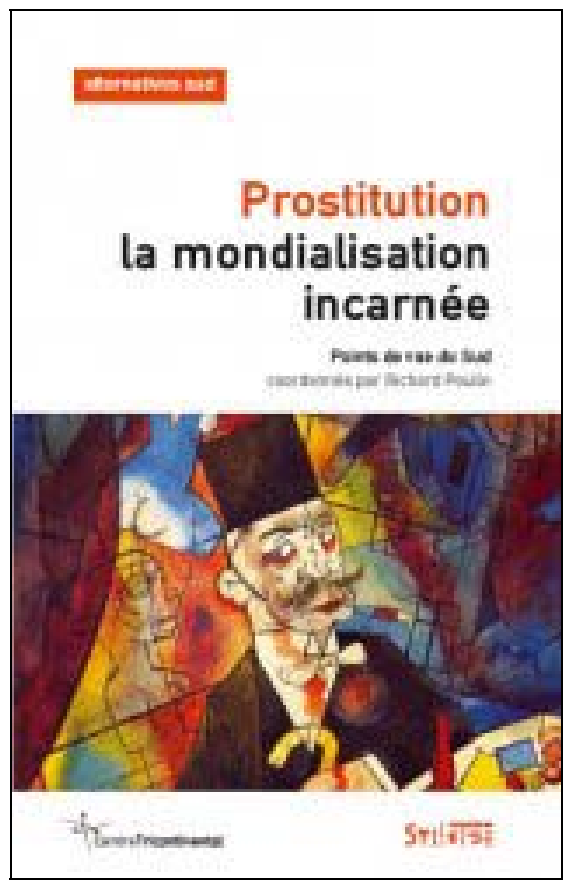

Un article publié dans la revue Alternatives Sud, vol. 12, no 3, automne 2005, pp. 7-29. "Éditorial". Numéro intitulé : "Prostitution, la mondialisation incarnée." Un numéro coordonné par Richard Poulin. Paris : Centre tricontinental et Syllepse. 


\section{Table des matières}

Introduction

1. La mondialisation et l'industrialisation du commerce du sexe sont deux phénomènes étroitement imbriqués.

2. Les politiques libérales participent à l'essor des industries du sexe.

3. La paupérisation de nombreuses régions du globe crée les conditions propices à toutes les formes de trafic, de traite et de prostitution d'êtres humains.

4. La mondialisation capitaliste a accentué l'inégalité de développement entre les pays, ce qui a produit une pression significative en faveur des migrations internationales.

5. L'industrialisation du commerce sexuel a induit le développement d'une production de masse de biens et de «services sexuels » qui a généré une division régionale et internationale du travail.

6. Malgré cela, la très grande majorité des analyses de la mondialisation capitaliste contemporaine ne prend pas en considération l'impact sur les sociétés et sur les rapports sociaux de sexe de l'industrie du commerce sexuel.

7. La prostitution est une activité traditionnelle du crime organisé et l'explosion des marchés sexuels est largement contrôlée par ce dernier.

8. La prostitution est fondée sur la violence. Elle se nourrit d'elle et l'amplifie.

9. Les femmes et les enfants des minorités sont victimes de l'industrie mondiale sexuelle d'une façon disproportionnée par rapport à leur proportion dans la population.

10. Le déploiement massif actuel de la prostitution est un effet, entre autres, de la présence de militaires engagés dans des guerres ou des occupations de territoire.

11. Entre un million et deux millions de mineurs rejoignent chaque année, dans le monde entier, les rangs des victimes du tourisme sexuel, c'est-à-dire de la prostitution organisée.

12. L'accumulation d'argent est le but du système dans sa totalité et, en particulier, du système proxénète qui domine et organise l'industrie de la prostitution.

13. La croissance effrénée des industries du sexe a pour effet une remise en cause des droits humains fondamentaux, notamment ceux des femmes et des enfants devenus des marchandises sexuelles.

14. Les valeurs libérales ont contaminé une partie importante de la gauche et du mouvement des femmes.

15. Il est vain de lutter contre la traite des êtres humains sans lutter contre le système prostitutionnel qui en est la cause.

Ouvrages cités 


\author{
Richard Poulin 1 \\ Sociologue, professeur titulaire, département de sociologie et d'anthropologie, \\ Université d'Ottawa. \\ ÉDITORIAL: \\ "Quinze thèses sur le capitalisme \\ et le système prostitutionnel mondial".
}

Un article publié dans la revue Alternatives Sud, vol. 12, no 3, automne 2005, pp. 7-29. "Éditorial". Numéro intitulé : "Prostitution, la mondialisation incarnée." Un numéro coordonné par Richard Poulin. Paris : Centre tricontinental et Syllepse.

\title{
Introduction
}

Retour à la table des matières

La mondialisation néo-libérale est le facteur dominant aujourd'hui dans l'essor de la prostitution et de la traite des femmes et des enfants aux fins de prostitution. Elle accroît les inégalités sociales et exploite les déséquilibres entre les hommes et les femmes qu'elle renforce singulièrement. Elle s'incarne dans une marchandisation des êtres humains et dans le triomphe de la vénalité sexuelle. Cette industrie est à la confluence des relations marchandes capitalistes et de l'oppression des femmes, deux phénomènes étroitement entremêlés. Construit autour de quinze thèses, ce texte tente schématiquement de mettre en évidence certains éléments d'analyse nécessaires à la compréhension de la mondialisation des industries du sexe.

1 Professeur de sociologie à l'Université d'Ottawa et auteur de La mondialisation des industries du sexe, Paris, Imago, 2005. 


\section{La mondialisation et l'industrialisation du commerce du sexe sont deux phénomènes étroitement imbriqués.}

Retour à la table des matières

La prostitution a pris un caractère de masse et a essaimé dans le monde entier. La pornographie est largement répandue dans les sociétés. Les chiffres donnent le tournis. Les gains de ces industries sont colossaux : en 2002, on estime que la prostitution engendre des revenus de 60 milliards $€$ et la pornographie 52 milliards $€$ (Dusch, 2002 : 109 et 101) ; le chiffre d'affaires des agences de tourisme sexuel œuvrant à partir du Web est évalué à 1 milliard $€$ par année; les profits de la traite à des fins de prostitution sont évalués entre 7,8 et 13,5 milliards $€$ par an (Konrad, 2002). Les êtres humains, principalement les femmes et les enfants, soumis à l'aliénation du commerce de leur sexe se comptent par dizaines de millions. Le nombre de personnes prostituées dans le monde est estimé, en 2001, à 40 millions (Healy, 2003). La clientèle croît à un rythme soutenu. Chaque année, environ 500000 femmes victimes de la traite aux fins de prostitution sont mises sur le marché de la vénalité sexuelle dans les pays de l'Europe de l'Ouest $2 ; 75 \%$ des femmes victimes de cette traite ont 25 ans et moins, et une proportion indéterminée d'entre elles, très importante, est constituée de mineures. Environ 4 millions de femmes et d'enfants sont victimes chaque année de la traite mondiale aux fins de prostitution. Au cours des années quatre-vingt-dix, en Asie du Sud-Est seulement, il y a eu trois fois plus de victimes de la traite à des fins de prostitution que dans l'histoire entière de la traite des esclaves africains. Selon Pino Arlacchi (cité par Demir, 2003) du Bureau des Nations Unies pour le contrôle des drogues et la prévention du crime, cette traite, qui a couru sur une période de 400 ans, aurait fait 11,5 millions de victimes, tandis que la traite aux fins de prostitution dans la seule région de l'Asie du Sud-Est a fait, en une décennie, 33 millions

2 Selon différentes sources, dont la Commission des droits de la femme et de l'égalité des chances du Parlement européen (2003) et Europol (2001). 
de victimes. Au cours des trois dernières décennies, les pays de l'hémisphère Sud ont connu une croissance vertigineuse de la prostitution et de la traite des femmes et des enfants à des fins prostitutionnelles. Depuis un peu plus d'une décennie, c'est également le cas des pays de l'ex-Union soviétique et de l'Europe de l'Est et centrale ainsi que des Balkans. Les êtres humains victimes de la traite mondiale à des fins de prostitution sont nettement plus nombreux que ceux qui sont l'objet d'un trafic aux fins d'exploitation domestique ou de maind'œuvre à bon marché. On estime que $90 \%$ des personnes victimes de la traite le sont à des fins de prostitution (Eriksson, 2004).

La tendance est à la prostitution d'enfants de plus en plus jeunes ainsi qu'à leur utilisation dans la pornographie. Que la prostitution d'enfants de douze ou quatorze ans soit légale ou non n'interfère en rien dans les problèmes éthiques soulevés par cette marchandisation sexuelle. L'industrie de la prostitution enfantine exploite 400000 enfants en Inde, 100000 aux Philippines, entre 200000 et 300000 en Thaillande, 100000 à Taiwan, entre 244000 et 325000 aux ÉtatsUnis. En Chine populaire, il y a entre 200000 et 500000 enfants prostitués. Entre 500000 et 2 millions d'enfants sont prostitués au Brésil. Quelque 35\% des personnes prostituées du Cambodge ont moins de 17 ans et $60 \%$ des Albanaises qui sont prostituées en Europe sont mineures ${ }^{3}$. Certaines études estiment qu'au cours d'une année, un enfant prostitué vend ses «services sexuels» à 2000 hommes (Robinson, 1998). Un rapport du Conseil de l'Europe estimait, en 1996, que 100000 enfants de l'Europe de l'Est se prostituaient à l'Ouest. À l'occasion du II $^{e}$ Congrès contre l'exploitation sexuelle des enfants à des fins commerciales, qui a eu lieu à Yokohama, au Japon, I'Unicef (2001) évaluait à plus d'un million le nombre d'enfants - des fillettes principalement - prostitués par l'industrie sexuelle. En 2004, les chiffres tournent autour de deux millions d'enfants. Aujourd'hui, au moins un million d'enfants sont prostitués en Asie du Sud-Est seulement; les

3 Chacune des données s'appuie sur des sources qu'il serait fastidieux de citer dans le cadre de ce texte. Les lecteurs peuvent consulter mon livre (Poulin, 2005) où elles sont détaillées. 
pays les plus touchés sont I'Inde, la Thaïlande, Taiwan et les Philippines.

L'industrie de la prostitution représente 5\% du PIB des Pays-Bas et de la Corée, entre 1 et $3 \%$ de celui du Japon et, en 1998, l'Organisation internationale du travail (OIT) a estimé que la prostitution représentait entre 2 et 14\% de l'ensemble des activités économiques de la Thaillande, de l'Indonésie, de la Malaisie et des Philippines (Lim, 1998). L'industrie pornographique est la troisième industrie en ordre d'importance du Danemark; elle a connu un développement fulgurant en Hongrie, devenu l'un des endroits prisés par les producteurs de films. Les industries sexuelles sont désormais des industries considérables - des multinationales pour certaines d'entre elles, cotées à la bourse - générant des profits fabuleux et des rentrées importantes en devises fortes, ce qui a un effet sur la balance des paiements des pays et donc sur leurs comptes courants; elles sont considérées comme vitales dans l'économie de plusieurs pays. La prostitution fait même partie de la stratégie de développement de certains États. En outre, sous l'obligation de remboursement de la dette, de nombreux États d'Asie ont été encouragés par les organisations internationales comme le Fonds monétaire internationale (FMI) et la Banque mondiale - qui ont offert des prêts importants - à développer leurs industries du tourisme et de divertissement. Dans chacun des cas, l'essor de ces secteurs a permis l'envolée de l'industrie du commerce sexuel. Dans certains pays, comme au Népal, les femmes et les enfants ont été mis directement sur les marchés régionaux ou internationaux (notamment en Inde et à Hong Kong), sans que le pays ne connaisse une expansion significative de la prostitution locale. Dans d'autres cas, comme en Thaillande, l'effet a été le développement simultané du marché local et des marchés régionaux et internationaux. Dans tous les cas, on observe que ces «marchandises» migrent des régions à faible concentration de capital vers les régions à plus forte concentration. Ainsi, par exemple, on estime que depuis dix ans, 200000 femmes et jeunes filles du Bangladesh ont été victimes de la traite à des fins de prostitution vers le Pakistan, de 20000 à 30000 personnes prostituées de Thaillande sont d'origine birmane et 150000 personnes prostituées en 
provenance des Philippines, de Taiwan, de Thaillande et de Russie ont été installées au Japon.

\section{Les politiques libérales participent à l'essor des industries du sexe}

Retour à la table des matières

Avec le triomphe des valeurs libérales dans le processus actuel de la mondialisation, le sexe tarifé ainsi que sa représentation, la pornographie, ont connu, dans les dernières décennies, une normalisation. La soumission aux règles du marché et aux lois libérales contractuelles d'échange entraîne une acceptation de plus en plus étendue de la prostitution. Elle est désormais, pour un nombre important d'États et d'organisations, un «métier comme un autre», un simple " travail du sexe» et même un «droit» ou une «liberté ». Depuis le début du nouveau millénaire, un certain nombre d'États a réglementé (légalisé) la prostitution (les Pays-Bas, l'Allemagne, la Suisse, l'Australie, la Nouvelle-Zélande, etc.). Au nom de l'« autonomie » des personnes et du droit de « contrôler son propre corps » est défendu le « droit» à la prostitution et à la traite des femmes aux fins de prostitution. Cette idéologie libérale s'est imposée peu à peu. Pendant longtemps, elle n'a pas semblé normale, morale ou «naturelle comme elle peut être perçue maintenant. Il a fallu des changements profonds et un ensemble de conditions propices à sa formulation en tant que "liberté ». Ces changements tiennent tout autant à la croissance des industries sexuelles qu'à la mondialisation néo-libérale, deux phénomènes étroitement imbriqués. Jamais dans l'histoire, la vénalité sexuelle n'a été aussi ample, profonde et banalisée. Les bouleversements qu'elle entraîne sont radicaux pour le tissu social et dans les mentalités. On assiste à la prostitutionnalisation de régions entières du globe et à une pornographisation des imaginaires sociaux, non seulement des systèmes de représentations, mais aussi de certaines façons de penser et d'agir.

La légalisation (réglementation) de l'industrie de la prostitution, proxénétisme y compris, a pour effet d'engendrer une croissance notable des industries du sexe et, par conséquent, entraîne une expan- 
sion de la traite à des fins de prostitution. Les Pays-Bas sont un bon indicateur de l'expansion de l'industrie sexuelle et de la croissance de la traite à des fins de prostitution: 2500 personnes prostituées en 1981, 10000 en 1985, 20000 en 1989 et 30000 en 2004. Il y a 2000 bordels dans le pays et au moins 7000 lieux voués au commerce du sexe : $80 \%$ des personnes prostituées sont d'origine étrangère et $70 \%$ d'entre elles dépourvues de papiers, ayant été victimes de la traite. En 1960, 95\% des prostituées des Pays-Bas étaient néerlandaises, en 1999 , elles ne sont plus que $20 \%$. Dans ce pays, la légalisation devait mettre fin à la prostitution des mineurs, or l'Organisation pour les Droits de l'enfant, dont le siège est à Amsterdam, estime que le nombre de mineurs qui se prostituent est passé de 4000 en 1996 à 15000 en 2001, dont au moins 5000 sont d'origine étrangère. Durant la première année de la légalisation néerlandaise, les industries du ont connu une croissance sexe de 25\% (Daley, 2001). Au Danemark, au cours de la dernière décennie, le nombre de personnes prostituées d'origine étrangère, victimes de la traite, a été multiplié par dix. En Autriche, $90 \%$ des personnes prostituées sont originaires d'autres pays, et en Italie, les ressortissantes de l'étranger constituent entre 67 et $80 \%$ des personnes prostituées (Covre et Paradiso, 2000). En Allemagne, 75 à $85 \%$ des personnes prostituées sont d'origine étrangère. En 2003, on estime, en Grèce, à 20000 les victimes de la traite aux fins de prostitution par année, tandis qu'elles étaient 2100 par année au début de la décennie précédente. En dix ans, de 1990 à 2000, 77500 jeunes femmes étrangères ont été la proie des trafiquants. Ces jeunes femmes, souvent des mineures, ont un prix d'achat sur les marchés balkaniques de $500 €$.. Il y a dix ans, le nombre de personnes prostituées d'origine grecque était estimé à 3400 ; aujourd'hui, leur nombre reste plus ou moins le même, mais avec l'explosion de l'industrie prostitutionnelle, le nombre de personnes prostituées d'origine étrangère a été multiplié par dix (Mitralias, 2003). LES POLITIQUES GOUVERNEMENTALES SONT UN FACTEUR DÉCISIF DANS LA PROLIFÉRATION DES INDUSTRIES PROSTITUTIONNELLES ET DE LA TRAITE QUI EN EST UN COROLLAIRE AINSI QUE DANS LEUR RENTABILITÉ. 


\section{La paupérisation de nombreuses régions du globe crée les conditions propices à toutes les formes de trafic, de traite et de prostitution d'êtres humains.}

\section{Retour à la table des matières}

Les plus touchés proviennent principalement des pays du Sud et de l'Est. À l'échelle de ces régions, le bouleversement des structures sociales dû au triomphe de l'économie capitaliste néo-libérale affecte grandement les zones rurales, pousse aux migrations vers les villes, favorise l'économie informelle, notamment les industries du sexe, et les déstructurations sociales. De même, l'extension de l'économie de marché et la croissance des inégalités sociales, renforcées par les plans d'ajustement structurel, les endettements importants des États ainsi que la financiarisation de l'économie sont loin d'exclure ou de marginaliser ses victimes. La mondialisation tire même avantage à « les produire » pour son plus grand profit. Les laissés-pour-compte - largement des femmes et des enfants - sont en réalité « la source des rentes les plus fortes de l'économie mondialisée » (Maillard, 2001 : $60)$.

\section{La mondialisation capitaliste a accentué l'inégalité de développement entre les pays, ce qui a produit une pression significative en faveur des migrations internationales.}

Cette mondialisation se caractérise par une féminisation de plus en plus importante des migrations. La Division de la population des $\mathrm{Na}-$ tions Unies estime que le nombre total de femmes vivant hors de leur pays de naissance équivaut à $48 \%$ de l'ensemble des migrants. La mondialisation se traduit par la féminisation de la pauvreté : sur le 1,3 milliard de personnes vivant dans la pauvreté dite absolue, $70 \%$ sont des femmes. Parallèlement à l'essor de la prostitution locale liée aux migrations de la campagne vers les villes, des centaines de milliers de 
jeunes femmes sont transportées vers les centres urbains du Japon, de l'Europe de l'Ouest et de l'Amérique du Nord pour «offrir » des « services sexuels ». Là où l'industrie de la prostitution est très développée, y compris dans les pays dépendants, des circuits de traite mondiaux sont mis en place dans un va-et-vient effarant : par exemple, en 1994, on estimait à 10000 le nombre de personnes prostituées originaires de l'Europe de l'Est opérant en Thaïlande. En contrepartie, les personnes prostituées thailandaises sont nombreuses à exercer dans les pays capitalistes dominants: en 1996, elles comptaient pour $60 \%$ des femmes de l'industrie du sexe japonaise. Ces réalités définissent les conditions et l'extension de la mondialisation capitaliste actuelle pour les femmes et les enfants victimes de l'industrie du sexe. Les prostituées étrangères se situent évidemment dans le bas de la hiérarchie prostitutionnelle, sont isolées socialement et culturellement, et exercent la prostitution dans les pires conditions possibles, tout en étant sujettes à différentes formes de violence, tant dans le quotidien prostitutionnel que dans leur transport d'un pays à l'autre ${ }^{4}$.

\section{L'industrialisation du commerce sexuel a induit le développement d'une production de masse de biens et de « services sexuels » qui a généré une division régionale et internationale du travail.}

\section{Retour à la table des matières}

Ces «biens » sont constitués d'êtres humains prostitués. Cette industrie, qui se déploie dans un marché mondialisé qui intègre à la fois le niveau local et le niveau régional, est devenue une force économique incontournable. La prostitution et les industries sexuelles qui lui sont connexes - les bars, les clubs de danseuses, les salons de massages, les maisons de production de pornographie, etc. - s'appuient sur une économie souterraine massive contrôlée par des proxénètes liés au crime organisé et bénéficient aux forces de police corrompues. Les

4 Parallèlement, nous assistons à une criminalisation des migrations, ce qui affecte particulièrement les femmes victimes de la traite à des fins de prostitution. Voir à ce sujet Poulin (2005: 76-78). 
chaînes hôtelières internationales, les compagnies aériennes et l'industrie touristique profitent largement de l'industrie du commerce sexuel. Les gouvernements eux-mêmes en bénéficient : en 1995, on a évalué que les revenus de la prostitution en Thaillande constituaient entre 59 et $60 \%$ du budget du gouvernement. Ce n'est pas sans raison que ce gouvernement faisait, en 1987, la promotion du tourisme sexuel en ces termes: " The one fruit of Thailand more delicious than durian [un fruit local], its young women. » (Santos, 1999)

\section{Malgré cela, la très grande majorité des analyses de la mondialisation capitaliste contemporaine ne prend pas en considération l'impact sur les sociétés et sur les rapports sociaux de sexe de l'industrie du commerce sexuel.}

Retour à la table des matières

Dans la très importante littérature produite sur le sujet, bien des aspects ont été examinés - privatisation, financiarisation, ajustement structurel, déréglementation, enrichissement et appauvrissement, croissance des inégalités, néo-libéralisme, réduction des budgets sociaux, programmes d'austérité, paradis fiscaux, etc. - mais rares sont les études intégrant dans la dynamique de la mondialisation l'essor des industries du sexe ${ }^{5}$. Pourtant, le processus de marchandisation des biens et des services, et plus particulièrement la commercialisation du vivant, y compris des corps et des sexes, ainsi que la monétarisation des relations sociales sont au cœur de l'actuelle accumulation capitaliste. Et « la marchandisation du vivant est exploitée par les mafias » (Passet et Liberman, 2002 : 38). Nombre d'opposants à la mondialisation néo-libérale et à l'extension du règne de la marchandise défendent la libéralisation des industries du sexe. Comme si le capitalisme n'avait pas récupéré le sexe et trouvé « vocation [...] à marchandiser le désir, notamment celui de la libération, et par là même à le récupérer

5 Une exception notable, ATTAC-France (2003) a développé une position politique abolitionniste sur la question. 
et à l'encadrer »(Boltanski et Chiapello, $2002: 226$ ). La mondialisation néo-libérale favorise la pénétration de la marchandise dans le domaine de mœurs et les révolutionne, ayant des effets considérables, mais mal connus, sur les codes sociaux ainsi que sur le psychisme humain et les rapports entre les hommes et les femmes. La « liberté sexuelle» est dorénavant « une valeur marchande et un élément des mœurs sociales ». «Le plaisir sous cette forme engendre la soumission 》 (Marcuse, 1968 : 108), particulièrement pour les femmes et les enfants transmutés en marchandises sexuelles.

\section{La prostitution est une activité traditionnelle du crime organisé et l'explosion des marchés sexuels est largement contrôlée par ce dernier.}

\section{Retour à la table des matières}

S'il en est ainsi, ce n'est pas parce que la prostitution est illégale ou prohibée. Dans les pays où la prostitution est légale (Allemagne, PaysBas, Suisse, Grèce), comme dans ceux où des bordels sont propriétés d'État (Turquie, Indonésie) ou dans les pays qui la reconnaissent comme une industrie vitale à l'économie nationale (Thaïlande, Philippines), le rôle du crime organisé reste fondamental dans l'organisation des marchés. C'est que la violence est décisive dans la production des «marchandises sexuelles» que sont les personnes prostituées. «En vingt jours, on peut briser n'importe quelle femme et la transformer en prostituée », raconte une responsable bulgare d'un foyer de réinsertion (citée par Chaleil, 2002 : 498). Le rapt, le viol, l'abattage - il existe de camps d'abattage ou de soumission non seulement dans les pays du Sud, mais également dans les Balkans, en Europe centrale et en Italie, où l'abattage est nommé « écolage» - la terreur et le meurtre ne cessent d'être des accoucheurs et des prolongateurs de cette industrie. Ils sont fondamentaux non seulement pour le développement des marchés, mais également pour la «fabrication» même des «marchandises», car ils contribuent à rendre les personnes prostituées «fonctionnelles» - cette industrie exigeant une disponibilité totale des corps. 
La criminalité financière et économique ainsi que toutes les autres formes de criminalités ne sont pas un phénomène marginal venant se greffer sur la mondialisation capitaliste: cette criminalité est consubstantielle, comme le souligne Jean de Maillard (2001), à la mondialisation néo-libérale et à son principe de la dérégulation. Les organismes financiers les plus « honorables » participent aux opérations de blanchiment, ce qui est en fait un mode de légalisation des profits criminels. Les États s'ils ne légalisent pas de telles activités en tirent néanmoins des bénéfices considérables. Le produit criminel brut est évalué à 15\% du commerce mondial (Passet et Liberman, 2002: 60). Actuellement, on assiste à une forte expansion des organisations criminelles se lançant dans la traite des êtres humains. En fait, au sein des secteurs d'activité de la criminalité organisée, la traite est le segment qui croît le plus rapidement.

\section{La prostitution est fondée sur la violence. Elle se nourrit d'elle et l'amplifie.}

\section{Retour à la table des matières}

Les violences exercées à l'endroit des personnes prostituées sont multiples et, souvent, innommables. La première violence est intrinsèque à la prostitution: la chosification et la marchandisation ont pour fonction la soumission des sexes à la satisfaction des plaisirs sexuels d'autrui. La deuxième lui est également inhérente : on devient une personne prostituée à la suite de violences sexuelles - selon différentes études, entre 80 et $90 \%$ des personnes prostituées en Occident ont été agressées sexuellement dans leur jeunesse 6 -, physiques, psychiques, sociales et économiques. La troisième est liée à l'expansion de la prostitution et de la traite à des fins de prostitution et à la dégradation consécutive des conditions dans lesquelles évoluent les personnes

6 Selon la plus récente enquête menée au Québec par l'anthropologue Rose Dufour (2005), 85\% des femmes prostituées ont subi des agressions sexuelles dans leur jeunesse. 
prostituées. L'extension du champ monétaire entraîne « la transformation en marchandise de ce qui n'est pas produit pour être marchandise » (Gauron, 2002 : 34). Ce processus de marchandisation s'opère au prix d'une tension et d'une violence considérables. Cela s'avère encore plus vrai dans la métamorphose de l'humain en marchandise. L'appropriation privée des corps, leur transmutation en marchandises et leur consommation, nécessite, en aval comme en amont, l'emploi de la force. La violence est constitutive de la marchandisation des êtres humains et de leur corps. Les méthodes de recrutement des proxénètes ne sont pas la simple addition de conduites privées et « abusives», mais s'insèrent dans un système structuré qui nécessite la violence. La brutalité d'un nombre important de clients dérive du fait que la transaction vénale leur confère une position de pouvoir. Une étude sur les personnes prostituées de rue en Angleterre établit que $87 \%$ d'entre elles ont été victimes de violence au cours des douze mois précédents : $43 \%$ d'entre elles souffrent de conséquences d'abus physique graves (Miller, 1995). Une recherche menée à Chicago a montré que $21,4 \%$ des femmes exerçant des activités d'escortes et de danseuses nues ont été violées plus de dix fois (Boulet, 2002). Une étude américaine menée à Minneapolis montre que $78 \%$ des personnes prostituées ont été victimes de viol par des proxénètes et des clients, en moyenne 49 fois par année: $49 \%$ ont été victimes d'enlèvement et transportées d'un État à un autre et $27 \%$ ont été mutilées (Raymond, 1999). Quelque $75 \%$ des escortes ont commis une tentative de suicide (Chester, 1994). Les femmes et les filles embrigadées dans la prostitution au Canada connaissent un taux de mortalité 40 fois supérieur à la moyenne nationale. L'âge moyen d'entrée dans la prostitution en Amérique du Nord est de 13 ou de 14 ans (Giobbe, 1992 ; John Howard Society of Alberta, 2001). Dans de telles conditions, peut-on soutenir qu'il y a vraiment une prostitution « libre», volontairement choisie?

Certains des bordels légaux du Nevada et du Nouveau-Mexique ont des enceintes grillagées, des chiens, des surveillants, comme s'ils n'étaient qu'un univers carcéral où les personnes prostituées sont en situation de détention ou d'esclavage. À Hambourg, les accès de certains quartiers réservés à la prostitution sont fermés par des chicanes. À Istanbul, l'entrée des complexes « bordeliers» que sont les ge- 
nelevs est sous surveillance. À Calcutta, des personnes prostituées s'offrent derrière les barreaux. En Thailande, des enfants sont sortis d'une cage pour le bénéfice des touristes sexuels. Le propriétaire d'une boîte de nuit, dans le nord-est de la Bosnie, s'est installé au milieu des champs de mines, avec une seule voie d'accès. Les personnes prostituées ne peuvent s'évader. Des guetteurs surveillent l'issue.

La prostitution soi-disant « libre » relève du libéralisme et non de la liberté. Entre 85 et $90 \%$ des personnes prostituées sont sous la coupe d'un proxénète ou d'un réseau de proxénètes 7 . À cause du déséquilibre des rapports de force et des discriminations systémiques, le droit libéral contractuel où deux personnes juridiquement égales passent un contrat, est, dans ce domaine comme ailleurs, un instrument d'asservissement et de dépendance des personnes. Les personnes soumises au pouvoir marchand masculin sont astreintes à ses règles et à son fonctionnement. Le droit d'une personne de se livrer à la prostitution et de permettre qu'une autre personne profite des revenus qu'elle en tire est, dans l'optique libérale, normalisé. La loi allemande légalisant la prostitution encourage la traite, via les agences internationales de mariage, en permettant à une conjointe née à l'étranger et mariée à un citoyen allemand de se prostituer et à son mari de vivre des fruits de sa prostitution. La mondialisation des marchés est, dans tous les textes internationaux ou européens, non seulement une valeur admise et commune, mais également une valeur à promouvoir. La marchandisation des êtres humains est autorisée, à la condition qu'elle ne soit pas «abusive », ou qu'elle ne soit pas « forcée » sous certaines conditions. Des formes légales de la traite sont permises, y compris dans les pays où la prostitution est illégale. De nombreux États délivrent des visas d'artistes pour les danseuses nues, recrutées en Europe de l'Est et en Asie du Sud-Est, et qui sont rapidement mises sur le marché prostitutionnel, quand les bars où elles évoluent ne sont pas eux-mêmes des lieux de prostitution. Les agences internationales de rencontre et de mariage avec les «beautés slaves» et les Asiatiques « exotiques» font des affaires d'or dans les pays capitalistes dominants.

7 Sur cette donnée, voir entre autres Chaleil (2002), Giobbe et al. (1990) et Hunter 1994). 
La prostitution n'est plus considérée comme une forme d'assujettissement du sexe féminin aux hommes, au système patriarcal, elle est désormais une un «droit» et une «liberté ». Les années quatre-vingt-dix ont été caractérisées par la légitimation de la marchandisation sexuelle des femmes et des enfants au profit du système prostitutionnel, au nom de la mise en cuvre de certaines modalités de sa régulation.

\section{Les femmes et les enfants des minorités sont victimes de l'industrie mondiale sexuelle d'une façon disproportionnée par rapport à leur proportion dans la population.}

\section{Retour à la table des matières}

C'est notamment le cas des minorités ethniques et des tribus de la province du Yunnan en Chine et des minorités ethniques en Thaillande du Nord et au Myanmar. Entre 1990 et 1997, environ 80000 femmes et enfants originaires de la région du Mékong ou appartenant aux ethnies vivant le long de la frontière entre la Thailande et le Myanmar ont été recrutés par l'industrie de la prostitution en Thaillande. Les personnes originaires de la minorité hongroise en Roumanie, de la minorité russe dans les pays baltes et des minorités tsiganes un peu partout en Europe de l'Est sont « sur-représentées » parmi les personnes prostituées dans leur propre pays ainsi qu'en Europe de l'Ouest. Les Autochtones du Canada et ceux de nombreux pays latino-américains sont également «sur-représentées » parmi les personnes prostituées de leurs pays respectifs. À l'échelle mondiale, les clients du Nord profitent de femmes et d'enfants du Sud et de l'Est, ainsi que des femmes et des enfants des minorités ethniques ou nationales. Au Sud lui-même les clients nationaux exploitent des femmes et des enfants de minorités nationales. 


\section{Le déploiement massif actuel de la prostitution} est un effet, entre autres, de la présence de militaires engagés dans des guerres ou des occupations de territoire.

\section{Retour à la table des matières}

La très importante industrie de la prostitution de l'Asie du Sud-Est a pris son envol grâce aux guerres de Corée et du Vietnam et au stationnement de troupes occidentales dans les pays limitrophes, notamment en Thaillande et aux Philippines. La croissance très importante de la prostitution locale a permis l'établissement de l'infrastructure nécessaire au développement du tourisme sexuel, grâce notamment à la disponibilité de la «main-d'œuvre » générée par la présence militai$\mathrm{re}^{8}$. Des loisirs plus importants, des facilités de communications et de déplacement vers l'étranger, la construction sociale, par la pornographie, d'une image exotique et sensuelle des personnes prostituées asiatiques, qui seraient, grâce à leur culture, sexuellement matures malgré leur jeune âge, et les politiques gouvernementales favorables au tourisme sexuel ont contribué à l'explosion de cette industrie. Dans les années quatre-vingt-dix, 18000 personnes prostituées coréennes étaient au service des 43000 militaires états-uniens stationnés dans ce pays. Aujourd'hui, 8500 femmes, originaires surtout des Philippines et de la Russie, sont victimes de la traite aux fins de prostitution pour les militaires états-uniens de Corée. Elles ont pu entrer au pays au moyen de visas de « divertissement » délivrés par le gouvernement à la suite de négociations avec l'association des propriétaires de bars des camptowns. On évalue qu'entre 1937 et 1945, l'armée japonaise d'occupation a utilisé entre 100000 et 200000 prostituées coréennes

8 Les États-Unis ont conclu une entente avec la Thaïlande en 1967 pour que le pays soit un lieu « de repos et de loisir» pour ses soldats. C'est un général de Royal Air Force thaïe qui a négocié cette entente qui a permis un afflux énorme de devises fortes dans l'économie du pays. Son épouse a dirigé la première agence de tours sexuels de la Thaillande pour les militaires américains. 
incarcérées dans des «bordels de réconfort». Quelques jours seulement après la défaite japonaise, l'Association pour la création de facilités récréatives spéciales, financée indirectement par le gouvernement nippon, ouvrait un premier bordel de réconfort pour les soldats états-uniens. À son point culminant, cette Association employait 70000 prostituées japonaises. L'Organisation internationale pour les migrations (OIM) évalue à 10000 le nombre de personnes prostituées clandestines en Bosnie. L'OIM estime que 250000 femmes et enfants de l'Europe de l'Est sont victimes de la traite via la Serbie et les États voisins, dont un grand nombre se retrouve dans les nouveaux protectorats internationaux de Bosnie et du Kosovo pour desservir soldats, policiers et membres des ONG. L'utilisation de «facilités récréatives » fait encore partie des politiques du Pentagone. Immédiatement après la première guerre contre l'Irak, les troupes étatsuniennes ont été envoyées en Thaïlande pour y prendre du «bon temps ».

\section{Entre un million et deux millions de mineurs rejoignent chaque année, dans le monde entier, les rangs des victimes du tourisme sexuel, c'est-à-dire de la prostitution organisée.}

\section{Retour à la table des matières}

Comme si la planète était devenue un immense lupanar. Le tourisme est un des secteurs très importants des économies des pays de l'Asie et du Pacifique. Il est au premier rang, en tant que secteur économique et source principale de devises, en Thaillande, en Australie et en Nouvelle-Zélande. Il se situe en deuxième position à Hong Kong, en Malaisie et aux Philippines et au troisième rang à Singapour et en Indonésie. En Nouvelle-Zélande, l'industrie touristique emploie plus de 200000 personnes. À Hong Kong, le tourisme emploie $12 \%$ de la main-d'œuvre et contribue pour environ $7 \%$ à l'économie. Le secteur du tourisme en Thailande concerne plus de 1,5 million d'emplois. À Singapour, la balance des paiements est attribuée par le FMI à l'excédent réalisé par le secteur du tourisme dont la part dans l'économie est d'environ $10 \%$. 
Tous les États sus-mentionnés sont des pays de destination de la traite des femmes et des enfants à des fins de prostitution. En 1998, on estimait à 200000 environ le nombre d'Allemands qui se rendaient à l'étranger chaque année pour avoir des relations sexuelles avec des enfants, leur préférence allant souvent aux fillettes au début de l'adolescence. Des agences allemandes spécialisées dans les rencontres et les mariages internationaux proposent également des mineures russes. En 2003, on évalue que $62 \%$ des enfants prostitués costaricains ont été la proie de touristes sexuels. Au moins 70 pages sur Internet présentent le Costa Rica comme un paradis sexuel. Un guide de voyage soulignait qu'il était aussi facile de "se procurer » une jeune Thaïlandaise que d'acheter un paquet de cigarettes» (Formoso, 2001). L'attrait que suscite la Thaïlande, le «pays du sourire», pour les étrangers n'a cessé de croître au cours des trois dernières décennies. En 1970, on comptabilisait 630000 visiteurs par an, en 1998, 7,8 millions. En 1995, le tourisme engendrait 7,1 milliards de dollars américains de recettes; il était la première source de devises étrangères et comptait pour $13 \%$ du PIB de la Thaillande. Le pays était la première destination récréative d'Asie du Sud-Est. Le sexe ratio des touristes en Thaillande présente un déséquilibre constant en faveur des hommes, qui constituent les deux tiers des visiteurs. Même si le sexe vénal avec les jeunes filles et garçons thailandais n'est pas nécessairement la principale motivation du séjour, peu se soustraient aux quartiers chauds « dont les guides de voyage présentent les attractions comme des curiosités locales, lorsqu'ils ne poussent pas à la consommation en donnant les "bonnes adresses" ou en insistant sur la facilité d'accès aux services sexuels» (Formoso, 2001). En 2001, 65\% des touristes qui visitaient le Cambodge étaient des hommes ; ce pays est connu pour la prostitution des enfants. Les touristes sexuels peuvent acheter des périples qui incluent vols internationaux, hébergement, fêtes privées, massages et la présence, chaque jour, de la fille de leur choix. Les tarifs pour une semaine : $7500 €$ pour Moscou ou $6000 €$ pour Bangkok et Pattaya (Dozier, 2004). La banalisation du tourisme à des fins de vénalité sexuelle est telle que le bordel australien Daily Planet a reçu, en 1991, le Victorian Tourism Award pour sa contribution à l'économie de la région. En 2001, à la faveur du Grand Prix de la Formule-1 de 
Hongrie, pour «bien desservir » les touristes, les autorités locales ont légalisé la prostitution pendant les trois jours de l'événement.

Le tourisme sexuel entraîne la prostitutionnalisation du tissu social : pour 5,4 millions de touristes sexuels par an en Thailande, on compte désormais 450000 clients locaux par jour (Jeffreys, 1999: 186-187). Désormais, 75\% des Thaïlandais sont clients.

Le tourisme sexuel n'est pas limité aux pays du Sud ou de l'Est. La Reeperbahn de Hambourg et les quartiers chauds d'Amsterdam et de Rotterdam sont des destinations bien connues des touristes sexuels. Les pays qui ont légalisé la prostitution ou qui la promeuvent sont devenus des lieux touristiques importants. C'est également à partir de ces pays que les ONG nationales militent au niveau européen et international pour faire reconnaître la prostitution comme un «travail sexuel ».

\section{L'accumulation d'argent est le but du système dans sa totalité et, en particulier, du système proxénète qui domine et organise l'industrie de la prostitution.}

\section{Retour à la table des matières}

L'argent est à la fois l'entremetteur entre le client et la personne prostituée et l'objectif de la transaction. Il confère pouvoir et toutepuissance aux proxénètes et aux clients et engendre la dévalorisation des personnes objets de la transaction sexuelle. Dans la société bourgeoise, les relations de pouvoir sont «à la fois intentionnelles et non subjectives», les rapports de domination et de sujétion sont immanents au « domaine où ils s'exercent » et « constitutifs de leur organisation ». Ces relations de pouvoir produisent la sexualité de nos sociétés. « Ironie de ce dispositif : il nous fait croire qu'il y va de notre "libération" », souligne Foucault (1976: 211). Caractérisée par la marchandisation, la vénalité sexuelle se concrétise donc dans l'objectivation, dans l'asservissement comme objet et dans la soumission à son moyen d'échange, l'argent, son appropriation exigeant à la fois aliénation et dessaisissement. 
La monétarisation des relations sociales « repose sur la marchandisation extensive des besoins sociaux, [et] la croissance actuelle implique une colonisation des rapports humains et de la culture par l'argent » (Perret, 2000). En effet, les rapports humains sont de plus en plus soumis à l'argent et à la marchandisation. La victoire du néolibéralisme dans les années quatre-vingt a permis une accélération de la soumission à la monétarisation des rapports sociaux. Cette accélération s'est traduite par un essor considérable des industries du sexe et par un discours, relevant du libéralisme le plus plat, qui légitime leurs activités. Cette monétarisation se produit en particulier au détriment des femmes et des enfants, devenus, pour des dizaines de millions d'entre eux, des «produits» sur les marchés sexuels, consommables et jetables après usage.

\section{La croissance effrénée des industries du sexe a pour effet une remise en cause des droits humains fondamentaux, notamment ceux des femmes et des enfants devenus des marchandises sexuelles.}

Retour à la table des matières

Le statut des femmes et des enfants a même gravement régressé. Désormais, dans de nombreux pays dépendants ainsi que dans ceux de l'ex-bloc soviétique, sous l'impact des politiques d'ajustement structurel et de l'économie de marché, les femmes et les enfants sont devenus de nouvelles matières brutes (new raw resources dans la littérature anglaise) exploitables et exportables dans le cadre du développement du commerce national et international. Du point de vue de leurs possesseurs, ces femmes et ces enfants se caractérisent par un double avantage. Il se traduit par la marchandisation non seulement des corps et des sexes, mais également par celle des femmes et des enfants vendus successivement à différents réseaux criminels proxénètes puis aux clients, d'où l'idée fréquente de l'apparition d'une nouvelle forme d'esclavage pour caractériser la traite dont sont victimes des millions de femmes et d'enfants. 
Le capitalisme néo-libéral trouve son expression achevée dans le domaine des industries du sexe. Ce régime d'accumulation étroitement lié aux dérégulations de la mondialisation renforce d'une façon considérable le système d'oppression des femmes et leur asservissement au plaisir d'autrui, au plaisir masculin. En réduisant les femmes à une marchandise susceptible d'être achetée, vendue, louée, appropriée, échangée ou acquise, la prostitution affecte les femmes en tant que groupe. Elle renforce l'équation établie par la société entre femme et sexe, réduisant les femmes à une humanité moindre et contribuant à les maintenir dans un statut inférieur partout dans le monde.

L'industrie du sexe est de plus en plus considérée comme une industrie du divertissement, et la prostitution comme un travail légitime. Elle est pourtant basée sur une violation systémique des droits humains et une oppression renforcée des femmes.

\section{Les valeurs libérales ont contaminé une partie importante de la gauche et du mouvement des femmes.}

\section{Retour à la table des matières}

Ce sont les sociaux-démocrates et les Verts allemands qui ont légalisé la prostitution. Au nom de la compétitivité des entreprises et des déficits budgétaires, ces mêmes partis remettent en cause certains des acquis sociaux. Sans tirer ici le bilan de cette gauche ${ }^{9}$ qui, au nom de valeurs qui relèvent davantage du libéralisme que du socialisme, s'est non seulement adaptée aux « contraintes " capitalistes, mais fait la promotion des bienfaits du marché, il faut souligner que son acceptation des valeurs libérales lui a permis de jouer, dans certains pays, un rôle actif dans la normalisation des industries du sexe au nom de la défense des «travailleuses du sexe» et du «droit à l'auto-

9 Voir sur ce bilan le livre de Serge Denis (2003). Ce texte est peu connu des Européens, mais mériterait de l'être davantage. 
détermination individuelle», dont le droit à la prostitution. Le mouvement des femmes est lui-même désormais divisé sur la question de la prostitution. Les organisations de femmes et les féministes libérales, qui défendent sa dépénalisation, s'appuient sur la distinction entre «prostitution volontaire» et «prostitution forcée». Pour Élisabeth Badinter (2002), par exemple, la prostitution s'intègre dans un « droit chèrement acquis depuis à peine trente ans [qui] appelle le respect de tous : la libre disposition de son corps ». La distinction entre prostitution « libre » et « forcée » lui permet de dénoncer le discours qui prétend que les personnes prostituées sont «les victimes de la logique économique libérale et de la domination masculine propre au patriarcat». Les qualifier de "victimes» serait admettre l'existence d'une oppression sociale structurelle, ce qui n'est plus le cas: « [L] e patriarcat [est] agonisant dans nos sociétés. »

Pour les défenseurs de la prostitution, certaines affirmations reviennent comme des leitmotive. Premièrement, la prostitution est, en général, un « travail volontairement choisi »; deuxièmement, la prostitution est l'équivalent d'un emploi de services, puisque c'est la simple vente d'un service sexuel; troisièmement, les restrictions légales à la prostitution constituent une violation des droits civiques, notamment celui de pouvoir librement choisir son emploi. Enfin, la légalisation mettrait fin à la stigmatisation du « métier», le normaliserait comme simple «travail du sexe » et conférerait des droits sociaux aux personnes prostituées. Sauf que dans les pays où la prostitution a été légalisée, les personnes prostituées qui s'enregistrent pour avoir accès à une protection sociale sont très minoritaires : $4 \%$ aux Pays-Bas, entre 5 et $8 \%$ en Allemagne, entre 6 et $10 \%$ à Vienne (Autriche), $7 \%$ à Athènes (Grèce), etc.

La prostitution, qu'elle soit légale ou illégale, comme les autres industries du sexe, n'est pas organisée pour les personnes prostituées, elle les marchandise et les monnaye. Elle est organisée par un système proxénète en faveur des clients. Où sont donc les proxénètes et les clients dans les propos des défenseurs de la prostitution? Au mieux, les clients n'apparaissent que comme parties contractantes de l'échange, que comme consommateurs. Ils ont le «droit» de consom- 
mer les personnes prostituées, puisque cela relève du droit contractuel bourgeois: c'est une entente conclue entre deux personnes consentantes (comme si la tierce personne, le proxénète, n'était jamais impliquée). Pourquoi ne pas défendre un autre droit du consommateur, celui de voir renouveler la marchandise périodiquement - la traite aux fins de prostitution ne sert-elle pas précisément à cela? En effet, cette traite ne leur pose pas problème, puisqu'elle est considérée, elle aussi, comme « volontaire » et est assimilée à la migration de « travailleuses du sexe». Ils ont peut-être le droit également à une qualité supérieure de marchandise? En Allemagne, toutes les entreprises de quinze employés et plus, y compris les bordels, doivent maintenant obligatoirement «embaucher » des apprentis sous peine de pénalités financières! Quelle personne sensée encouragerait une quelconque adolescente à suivre un apprentissage dans un Eros center?

Définir la prostitution ou la traite aux fins de prostitution par la contrainte ou l'absence de contrainte implique qu'il n'est plus nécessaire d'analyser la prostitution en tant que telle: son sens, ses mécanismes, ses liens avec le crime organisé, son inscription dans les relations marchandes et patriarcales, son rôle dans l'oppression des femmes, etc. La légitimation de la prostitution passe par cette opération de réduction libérale. C'est en 1993, que l'Union européenne et les organisations internationales ont commencé à utiliser l'expression «prostitution forcée ». Depuis, les documents internationaux et européens ne s'engagent à lutter que contre certaines formes de la traite à des «fins d'exploitation sexuelle». Avec la négation du lien entre la traite et la prostitution, source de la traite, les pays ayant légalisé la prostitution peuvent adhérer aux nouvelles conventions qui condamnent certaines formes de traite (du point de vue de la criminalité transnationale et non de la prostitution des femmes). Combattre uniquement la traite, c'est réprimer le transfert de personnes prostituées entre les pays et non pas lutter contre leur prostitution ${ }^{10}$. Cela est encore plus

10 L'Allemagne, les Pays-Bas et la Suisse font non seulement de la prostitution un «travail comme un autre», mais également la promotion de la « préférence nationale » sur le marché de la prostitution. Une citoyenne ou une personne étrangère en situation régulière (mariée à un citoyen du pays) a le « droit de se prostituer ». Aux autres est refusé ce «droit». Elles ne peuvent alors bénéficier 
vrai lorsque cela ne concerne que les formes les plus abusives de la traite et non la traite elle-même.

\section{Il est vain de lutter contre la traite des êtres humains sans lutter contre le système prostitutionnel qui en est la cause.}

Retour à la table des matières

L'officialisation institutionnelle (la légalisation) des marchés du sexe renforce les activités de l'organisation proxénète et du crime organisé. Cette consolidation, accompagnée d'un accroissement important des activités prostitutionnelles et de la traite, entraîne une dégradation non seulement de la condition générale des femmes et des enfants, mais aussi, en particulier, celle des personnes prostituées et des victimes de la traite à des fins de prostitution, ces dernières étant généralement criminalisées en tant qu'immigrantes clandestines.

La lutte contre la prostitution et la traite aux fins de prostitution s'inscrit dans l'objectif plus général de lutte pour l'égalité des femmes et des hommes. Cette égalité restera inaccessible tant que les hommes achètent, vendent et exploitent des femmes et des enfants en les prostituant.

L'abolitionnisme féministe représente une résistance à cette marchandisation sexuelle; il est un élément fondamental de la lutte contre le néo-libéralisme, la privatisation du vivant, la mondialisation capitaliste et le système proxénète planétaire. Cet abolitionnisme s'oppose à la monétarisation des rapports sociaux et à la marchandisation du sexe des êtres humains. Il est la seule position juridique, philosophique et

des droits sociaux accordés par l'État aux «travailleuses du sexe». Contre la concurrence des personnes prostituées étrangères, qui arrivent massivement sur le marché et qui « cassent les prix», s'élèvent des voix déplorant leur esclavage sexuel, leur prostitution «forcée » et exigeant leur « libération »! L'internationalisation des industries du sexe s'accompagne en ce début de nouveau millénaire d'une « nationalisation » du « droit à la prostitution »! 
politique qui peut permettre la contestation de l'ordre marchand et sexiste tel qu'il se déploie dans l'industrie mondialisée du commerce du sexe. Il est basé sur le caractère inaliénable du corps humain. L'abolitionnisme traditionnel vise l'« abolition » des règlements sur les personnes prostituées, et non l'abolition de la prostitution, et ne s'attaque pas à l'une des causes de la prostitution: les clients (la demande) ${ }^{11}$. Il n'a pas non plus développé les politiques sociales permettant aux personnes prostituées d'échapper au système prostitutionnel ${ }^{12}$. Cet abolitionnisme, qui fonde le système juridique de nombreux États, doit donc être repensé et réactualisé.

\section{Ouvrages cités}

Retour à la table des matières

ATTAC, (2003), Quand les femmes se heurtent à la mondialisation, Paris, Mille et une nuits.

AUDET, Élaine (2002), La prostitution: droits des femmes ou droit aux femmes? [en ligne], Montréal, Sisyphe, 26 septembre 2002 [site visité le 22 avril 2003],

http://sisyphe.org/article.php3?id_article=102.

BADINTER, Élisabeth (2002), «Rendons la parole aux prostituées», Le Monde, 31 juillet.

BOLTANSKI, Luc et Ève CHIAPELlo (2002), Le nouvel esprit du capitalisme, Paris, Gallimard.

11 La politique néo-abolitionniste de la Suède s'attaque à ceux qui profitent de la prostitution, clients y compris. La particularité de la nouvelle loi sur la prostitution est de s'inscrire dans une loi cadre plus importante, appelé en français « La Paix des femmes», qui concerne la violence faite aux femmes. La prostitution est assimilée à une violence à l'égard les femmes. Depuis l'adoption de la loi, la Suède est l'un des seuls pays à avoir connu une décroissance marquée de la prostitution sur son territoire et à avoir échappé à la traite des femmes et des enfants. Voir à ce sujet, Poulin (2005) et Ngalikpima (2005).

12 Une étude du Conseil du statut de la femme du Québec démontrait que « $92 \%$ des prostituées quitteraient la prostitution si elles le pouvaient». Citée par Au$\operatorname{det}(2002)$. 
BOULET, Elsa (2002), Rapport sur la prostitution à Chicago [en ligne], Paris, Les Pénélopes, octobre, [site visité le 27 février 2003],

http://www.penelopes.org/xarticle.php3?id_article=2296.

CHALEIL, Max (2002), Prostitution. Le désir mystifié, Paris, Parangon.

CHESTER Phillis (1994), Partiarchy. Notes of an Expert Witness, Monroe, Common Courage Press.

COMMISSION DES DROITS DE LA FEMME ET DE L'ÉGALITÉ DES CHANCES (2003), Communication aux membres. Objet: principales activités au cours de la cinquième législature, Parlement européen, Direction générale des commissions et délégations, 25 septembre, CMI505949FR.doc, PE 331.511/rev.

COVRE, Pia, avec Rosanna PARADISO (2000), Southern Regional Report. Parts I et II [en ligne], [site visité 3 avril 2003]

http://www.med.ic.ac.uk/divisions/60/europapnew/regional/southe rn regional.htm.

DALEY, Suzanne (2001), « New rights for Dutch prostitutes, but no gain », New York Times, August 12 : A1 et A4.

DENIS, Serge (2003), Social-démocratie et mouvements ouvriers. La fin de l'histoire?, Montréal, Boréal.

DEMIR, Jenna Shearer (2003), Trafficking of women for sexual exploitation: a gender-based well-founded fear? An examination of refugee status determination for trafficked prostituted women from CEE/CIS countries to Western Europe [en ligne], [site visité le 10 octobre 2003],

http://www.antislavery.org/homepage/traffic\%20news/shearerde mir 2004.pdf.

DOZIER, Marc (2004), «Les dessous du tourisme sexuel», Les Grands Reportages, $n^{\circ} 269$, juin.

DUFOUR, Rose (2005), Je vous salue... Le point zéro de la prostitution, Sainte-Foy, Multi-mondes.

DusCH, Sabine (2002), Le trafic d'êtres humains, Paris, Presses Universitaires de France.

ERIKSSON, Marianne (2004), Rapport sur les conséquences de l'industrie du sexe dans I'Union européenne [en ligne], Montréal, Sisyphe, 16 mai [site visité le 12 octobre 2004],

http://sisyphe.org/article.php3?id_article=1104. 
EUROPOL (2001), Threat Assessment on Russian Organised Crime, File $n^{\circ}$ 2520-31, June.

FORMOSO, Bernard (2001), « Corps étrangers. Tourisme et prostitution en Thailande », Anthropologie et Sociétés, vol. 25, n², 2001 : 55-70.

FouCAULT, Michel (1976), Histoire de la sexualité. I. La volonté de savoir, Paris, Gallimard.

GaURoN, André (2002), L'empire de l'argent, Paris, Desclée de Brouwer.

GIOBBE E., (1992), « Juvenil Prostitution: Profile of Recruitment », Child Trauma I: Isssues \& Research, New York, Garaland Publishing.

GiobBe, E., M. HaRRIGAN, J. Ryan et D. GaMACHE (1990), Prostitution. A Matter of Violence against Women, WHISPER, Minneapolis, $M N$.

HeAly, Grainne (2003), Presentation by Grainne Healy Chair of EWL's Observatory on Violence against Women [en ligne], EWL Seminar on Trafficking and Prostitution - side event at CSW, 17 mars, [site visité le 20 mars 2003],

http://www.womenlobby.org/Document.asp?DocID=566\&tod=4931.

HUNTER, S. K. (1994), «Prostitution is cruelty and abuse to women and children », Michigan Journal of Gender and Law, $n^{\circ} 1: 1-14$.

JEFFREYS, Sheila (1999), « Globalizing sexual exploitation. Sex tourism and the traffic in women », Leisure Studies, vol. $18, n^{\circ} 3$, July: 179-196.

KONRAD, Helga (2002), Trafficking in Human Beings. The Ugly Face of Europ, [en ligne], European Conference on Preventing and Combating Trafficking in Human Beings Global Challenge for the 21st Century, 18-20 September, [site visité le 22 mars 2004],

http://www.belgium.iom.int/STOPConference/Conference\%2OPape rs $/ 11 . \% 20$ Konrad\%20\%20Paper\%20EC-IOM\%20STOP\%20Conf.doc.

JOHN HOWARD SOCIETY OF ALBERTA (2001), Prostitution [en ligne], Research and Publication, [site visité le 6 mars 2003],

http:www.johnhoward.ab.ca/PUB/prositu.htm.

LIM, Lin Lam (1998), The Sex Sector. The Economic and Social Bases of Prostitution in Southeast Asia, Geneva, OIT.

LOUIS, Marie-Victoire (1997), «Le corps humain mis sur le marché », Le Monde diplomatique, mars, 1997, p. 8. 
MAILLARD, Jean de (2001), Le marché fait sa loi, Paris, Mille et une nuits.

MARCUSE, Herbert (1968), L'homme unidimensionnel, Paris, Seuil.

MILLER, J. (1995), «Gender and power on the streets: Street prostitution in the era of crack cocaine », Journal of Contemporary Ethnography, vol. 23, $n^{\circ} 4: 427-452$.

MITRALIAS, Sonia (2003), La traite des femmes en Grèce, un véritable enjeu de civilisation [en ligne], Paris, Les Pénélopes, 12 novembre, [site visité le 3 avril 2004],

http://www.penelopes.org/xarticle.php3?id_article=4460.

NGaLIKPIMA, Matiada, dir., (2005), L'esclavage sexuel: un défi à l'Europe, Paris, Éditions de Paris et Fondation Scelles.

PASSET, René et Jean LIBERMAN (2002), Mondialisation financière et terrorisme, Montréal, Écosociété.

PERRET, Bernard (2000), «De la critique du marché à celle de l'argent », Esprit critique, vol. $2 n^{\circ} 10$, octobre.

POULIN, Richard (2005), La mondialisation des industries du sexe, Paris, Imago.

RAYMOND, Janice (1999), Health Effects of Prostitution [en ligne], The Coalition Against Trafficking in Women, Kingston, University of Rhode Island, [site visité le ler mars 2001],

http://www.uri.edu/artsci/wms/Huques/mhvhealth.htm.

ROBINSON, L. N. (1998), The Globalization of Female Child Prostitution [en ligne], Bloomington, Indiana University, [site visité le 4 juin 1999], http ://www.law.indiana.edulglsj/ vol5/no1/robinson.html.

SANTOS, Aida F. (1999), Globalization, Human Rights and Sexual Exploitation [en ligne], Kingston, University of Rhode Island, The Coalition Against Trafficking in Women, [site visité le ler mars 2001], http :www.uri.edu/artsci/wms/hugues/ mhvglo.html.

UNICEF (2001), L'Unicef demande l'élimination de l'exploitation sexuelle des enfants à des fins commerciales [en ligne], New York, Unicef [site visité le $1^{\text {er }}$ mars 2002],

http://www.unicef.org/french/newsline/pr/2001/01pr97fr.htm.

Fin du texte 